\title{
NORMALISASI POSITIF SISTEM SINGULAR DISKRIT
}

\author{
RAKIB HABLULLAH, ZULAKMAL \\ Program Studi Matematika, \\ Fakultas Matematika dan Ilmu Pengetahuan Alam, Universitas Andalas, \\ Kampus UNAND Limau Manis Padang, Indonesia, \\ email : rakibhablullah15@gmail.com
}

\begin{abstract}
Abstrak. Suatu sistem singular diskrit dikatakan mempunyai solusi jika syarat regularitas terpenuhi. Pada tugas akhir ini, dinyatakan bahwa sistem singular diskrit dapat dinormalkan jika terdapat suatu vektor $\mathbf{w}(t)=-\mathcal{K} \mathbf{y}(t+1)$, sedemikian sehingga $\operatorname{det}(F+T \mathcal{K}) \neq 0$. Sistem singular diskrit normal dikatakan positif jika syarat matriks $\mathcal{K} \in \mathbb{R}^{m \times n}$ sedemikian sehingga $\operatorname{det}(F+T \mathcal{K}) \neq 0$ dan solusi dari sistem tersebut adalah positif.
\end{abstract}

Kata Kunci: singular, monomial, rank, diskrit, normal, positif

\section{PENDAHULUAN}

Diberikan sistem linier berikut:

$$
F \mathbf{y}(t+1)=S \mathbf{y}(t)+T \mathbf{w}(t), t \in \mathbb{Z}_{+},
$$

dimana $F, S \in \mathbb{R}^{n \times n}, T \in \mathbb{R}^{n \times m}, \mathbf{y}(t) \in \mathbb{R}^{n}, \mathbf{w}(t) \in \mathbb{R}^{m}$.

Sistem (1.1) dengan $\operatorname{rank}(F)<n$ mempunyai solusi jika $\operatorname{det}(\lambda F-S) \neq 0$ untuk suatu $\lambda \in \mathbb{C}$, dimana $\mathbb{C}$ menyatakan himpunan bilangan kompleks. Dalam hal $\operatorname{det}(\lambda F-S) \neq 0$ untuk suatu $\lambda \in \mathbb{C}$, sistem (1.1) disebut sebagai sistem singular reguler.

Dalam beberapa situasi kadang-kadang diperlukan upaya untuk menormalkan sistem (1.1). Dalam [6] dinyatakan bahwa sistem (1.1) dapat dinormalkan jika terdapat suatu vektor $\mathbf{w}(t)=-\mathcal{K} \mathbf{y}(t+1)$, untuk suatu $\mathcal{K} \in \mathbb{R}^{m \times n}$ sedemikian sehingga $\operatorname{det}(F+T \mathcal{K}) \neq 0$. Dengan $\mathbf{w}(t)=-\mathcal{K} \mathbf{y}(t+1)$, sistem (1.1) dapat diubah menjadi

$$
(F+T \mathcal{K}) \mathbf{y}(t+1)=S \mathbf{y}(t)
$$

Jelas bahwa jika $\operatorname{det}(F+T \mathcal{K}) \neq 0$, maka sistem (1.2) menjadi normal, yaitu

$$
\mathbf{y}(t+1)=(F+T \mathcal{K})^{-1} S \mathbf{y}(t)
$$

Dalam makalah ini akan dibicarakan masalah normalisasi positif dari sistem (1.1) yaitu bagaimana syarat matriks $\mathcal{K} \in \mathbb{R}^{m \times n}$ sedemikian $\operatorname{sehingga} \operatorname{det}(F+$ $T \mathcal{K}) \neq 0$ dan solusi dari sistem (1.3) adalah positif, yaitu $\mathbf{y}(t) \in \mathbb{R}_{+}^{n}$. 
64 Rakib Hablullah, Zulakmal

\section{LANDASAN TEORI}

\subsection{Teori Matriks}

Definisi 2.1. [1] Suatu matriks $A \in \mathbb{R}_{+}^{n \times n}$ dikatakan matriks monomial jika dalam setiap baris dan kolom dari A terdapat tepat satu entri yang tak nol dan entri-entri lainnya adalah nol.

Definisi 2.2. [7] Suatu matriks $A \in \mathbb{R}^{n \times n}$ dikatakan monoton jika $A^{-1}$ ada dan $A^{-1} \in \mathbb{R}_{+}^{n \times n}$.

Teorema 2.3. [7] Jika $A \in \mathbb{R}^{n \times n}$ maka $A^{-1} \in \mathbb{R}_{+}^{n \times n}$ jika dan hanya jika $A$ matriks monomial.

Contoh 2.4. Diberikan matriks

$$
A=\left[\begin{array}{lll}
4 & 0 & 0 \\
0 & 0 & 3 \\
0 & 2 & 0
\end{array}\right] .
$$

Diperoleh invers matriks $A$ adalah

$$
A^{-1}=\left[\begin{array}{ccc}
\frac{1}{4} & 0 & 0 \\
0 & 0 & \frac{1}{2} \\
0 & \frac{1}{3} & 0
\end{array}\right] .
$$

Karena $A \in \mathbb{R}^{n \times n}$ dan $A^{-1}$ ada, dimana $A^{-1} \in \mathbb{R}_{+}^{n \times n}$ maka $A$ dikatakan monoton dan $A$ adalah matriks monomial.

Definisi 2.5. [3]

(i). Dua matriks $M \in \mathbb{R}_{+}^{n \times n}$ dan $N \in \mathbb{R}_{+}^{n \times n}$ dikatakan ekuivalen positif, jika ada dua matriks monomial $B$ dan $A$ sedemikian sehingga

$$
N=A M B \text {. }
$$

(ii). Diberikan matriks $N \in \mathbb{R}_{+}^{n \times n}$ dengan $\operatorname{rank}(N)=r$. Matriks $N$ dikatakan $r$ monomial jika ia ekuivalen positif dengan matriks

$$
\left[\begin{array}{cc}
N_{r} & O \\
O & O
\end{array}\right]
$$

dimana $N_{r}$ adalah monomial.

Proposisi 2.6. [3]

(i). Matriks $N$ adalah r-monomial jika dan hanya jika $N$ mempunyai $(n-r)$ baris dan $(n-r)$ kolom yang semua entri-entrinya sama dengan nol dan $r$ baris dan $r$ kolom dengan hanya satu entri tak nol.

(ii). Jika matrik $N$ adalah r-monomial, maka ia ekuivalen positif dengan matriks

$$
\left[\begin{array}{ll}
I_{r} & O \\
O & O
\end{array}\right]
$$




\subsection{Sistem Linier Normal Positif}

Perhatikan sistem linier diskrit normal berikut ini:

$$
\mathbf{y}(t+1)=S \mathbf{y}(t)+T \mathbf{w}(t), t \in \mathbb{Z}_{+},
$$

dimana $S \in \mathbb{R}^{n \times n}, T \in \mathbb{R}^{n \times m}, \mathbf{y}(t) \in \mathbb{R}^{n}, \mathbf{w}(t) \in \mathbb{R}^{m}$.

Solusi dari sistem (2.2) diberikan sebagai berikut [9] :

$$
\mathbf{y}(t)=S^{t} \mathbf{y}(0)+\sum_{k=0}^{t-1} S^{t-k-1} T \mathbf{w}(k) .
$$

Definisi 2.7. [9] Sistem (2.2) dikatakan positif jika untuk setiap syarat awal $\mathbf{y}_{0} \in$ $\mathbb{R}_{+}^{n}$ dan untuk setiap vektor $\mathbf{w}(\mathbf{k}) \in \mathbb{R}_{+}^{m}$ maka solusi dari sistem adalah nonnegatif untuk setiap $t \in \mathbb{Z}_{+}$, yaitu $\mathbf{y}(t) \in \mathbb{R}_{+}^{n}$.

Teorema 2.8. [9] Sistem (2.2) adalah positif jika dan hanya jika

$$
S \in \mathbb{R}_{+}^{n \times n} \text { dan } T \in \mathbb{R}_{+}^{n \times m} .
$$

Contoh 2.9. Diberikan sistem (2.2) dengan

$$
S=\left[\begin{array}{ll}
1 & 2 \\
0 & 1
\end{array}\right], T=\left[\begin{array}{l}
1 \\
0
\end{array}\right]
$$

Jika diberikan $\mathbf{y}(0) \in \mathbb{R}_{+}^{2}$ dan $\mathbf{w}(t) \in \mathbb{R}_{+}$dimana $t>0$, maka solusi dari sistrim linier diskrit tersebut adalah

$$
\left[\begin{array}{l}
y_{1}(t) \\
y_{2}(t)
\end{array}\right]=\left[\begin{array}{ll}
1 & 2 \\
0 & 1
\end{array}\right]^{t}\left[\begin{array}{l}
y_{1}(0) \\
y_{2}(0)
\end{array}\right]+\sum_{k=0}^{t-1}\left[\begin{array}{ll}
1 & 2 \\
0 & 1
\end{array}\right]^{t-k-1}\left[\begin{array}{l}
1 \\
0
\end{array}\right][w(k)] \in \mathbb{R}_{+}^{2}
$$

\section{Pembahasan}

Seperti yang telah diketahui bahwa sistem (1.1) dikatakan dapat dinormalkan jika terdapat suatu vektor $\mathbf{w}(t)=-\mathcal{K} \mathbf{y}(t+1), t \in \mathbb{Z}_{+}$, untuk suatu $\mathcal{K} \in \mathbb{R}^{m \times n}$ sedemikian sehingga $\operatorname{det}(F+T \mathcal{K}) \neq 0$.

Proposisi 3.1. [4] Sistem (1.1) dapat dinormalkan jika dan hanya jika rank $[F T]=n$.

Asumsikan bahwa matriks $F$ ekuivalen positif dengan suatu matriks $r$-monomial sebutlah $N_{r}$, maka terdapat matriks monomial $A$ dan $B$ dimana $A, B \in \mathbb{R}_{+}^{n \times n}$ sedemikian sehingga

$$
A F B=\left[\begin{array}{cc}
N_{r} & O \\
O & O
\end{array}\right] .
$$

Misalkan

$$
A T=\left[\begin{array}{l}
T_{1} \\
T_{2}
\end{array}\right]
$$


Maka syarat rank $[F T]=n$ haruslah ekuivalen dengan matriks $T_{2}$ yang mempunyai full row rank, yaitu $(n-r)$. Hal ini dapat dibuktikan sebagai berikut

$$
\begin{aligned}
& \operatorname{rank}[F T]=n \Leftrightarrow \operatorname{rank}[A F B A T]=n \\
& \Leftrightarrow \operatorname{rank}\left[\begin{array}{ccc}
N_{r} & O & T_{1} \\
O & O & T_{2}
\end{array}\right]=n .
\end{aligned}
$$

Karena $\operatorname{rank}\left(N_{r}\right)=r$, maka mestilah $\operatorname{rank}\left(T_{2}\right)=(n-r)$.

Teorema 3.2. [3] Diberikan sistem (1.1) dengan matriks $F$ dan $T$ diberikan oleh (3.1) dan (3.2), dimana rank $\left(T_{2}\right)=(n-r)$ dan $S \in \mathbb{R}_{+}^{n \times n}$. Maka terdapat matriks $\mathcal{K} \in \mathbb{R}^{m \times n}$ sedemikian sehingga $\operatorname{det}(F+T \mathcal{K}) \neq 0$ dan sistem (1.3) adalah positif jika

a. $\left(T_{2} T_{2}^{T}\right)^{-1} \in \mathbb{R}_{+}^{(n-r) \times(n-r)}$;

b. $\left(T_{1} T_{2}^{T}\right) \in \mathbb{R}_{-}^{r \times(n-r)}$;

c. $\left(T_{2} T_{2}^{T}\right)^{-1} T_{2} \in \mathbb{R}_{+}^{(n-r) \times m}$;

d. $T_{1}-T_{1} T_{2}^{T}\left(T_{2} T_{2}^{T}\right)^{-1} T_{2} \in \mathbb{R}_{+}^{r \times m}$

Bukti. Misalkan $\mathcal{K}=\left[O T_{2}^{T}\right] B^{-1}$, maka

$$
\begin{aligned}
& F+T \mathcal{K}=A^{-1}\left[\begin{array}{cc}
N_{r} & O \\
O & O
\end{array}\right] B^{-1}+A^{-1} A T \mathcal{K} \\
& =A^{-1}\left[\begin{array}{cc}
N_{r} & O \\
O & O
\end{array}\right] B^{-1}+A^{-1}\left[\begin{array}{l}
T_{1} \\
T_{2}
\end{array}\right]\left[\begin{array}{ll}
O & T_{2}^{T}
\end{array}\right] B^{-1} \\
& =A^{-1}\left(\left[\begin{array}{cc}
N_{r} & O \\
O & O
\end{array}\right]+\left[\begin{array}{c}
T_{1} \\
T_{2}
\end{array}\right]\left[\begin{array}{ll}
O & T_{2}^{T}
\end{array}\right]\right) B^{-1} \\
& =A^{-1}\left(\left[\begin{array}{cc}
N_{r} & O \\
O & O
\end{array}\right]+\left[\begin{array}{cc}
O & T_{1} T_{2}^{T} \\
O & T_{2} T_{2}^{T}
\end{array}\right]\right) B^{-1} \text {. } \\
& =A^{-1}\left[\begin{array}{cc}
N_{r} & T_{1} T_{2}^{T} \\
O & T_{2} T_{2}^{T}
\end{array}\right] B^{-1} \text {. }
\end{aligned}
$$

Karena $\operatorname{rank}\left(T_{2}\right)=n-r$ maka $(F+T \mathcal{K})$ adalah nonsingular, sehingga $\operatorname{det}(F+T \mathcal{K} \neq 0)$. Selanjutnya, akan ditunjukan bahwa sistem (1.3) adalah positif. Perhatikan bahwa

$$
\begin{aligned}
(F+T \mathcal{K})^{-1} & =\left(A^{-1}\left(\left[\begin{array}{ll}
N_{r} & O \\
O & O
\end{array}\right]+\left[\begin{array}{ll}
O & T_{1} T_{2}^{T} \\
O & T_{2} T_{2}^{T}
\end{array}\right]\right) B^{-1}\right)^{-1} \\
& =\left(A^{-1}\left[\begin{array}{cc}
N_{r} & T_{1} T_{2}^{T} \\
O & T_{2} T_{2}^{T}
\end{array}\right] B^{-1}\right)^{-1} \\
& =B\left[\begin{array}{cc}
N_{r} & T_{1} T_{2}^{T} \\
O & T_{2} T_{2}^{T}
\end{array}\right]^{-1} A .
\end{aligned}
$$


sehingga diperoleh

$$
\begin{aligned}
& (F+T \mathcal{K})^{-1}=B\left[\begin{array}{cc}
N_{r}^{-1}-N_{r} T_{1} T_{2}^{T}\left(T_{2} T_{2}^{T}\right)^{-1} \\
O & T_{2} T_{2}^{T}
\end{array}\right] A \\
& =B\left[\begin{array}{cc}
N_{r} & O \\
O & I
\end{array}\right]\left[\begin{array}{cc}
I & -T_{1} T_{2}^{T}\left(T_{2} T_{2}^{T}\right)^{-1} \\
O & \left(T_{2} T_{2}^{T}\right)^{-1}
\end{array}\right] A
\end{aligned}
$$

Dari kondisi (a) dan (b) diperoleh $(F+T \mathcal{K})^{-1} \in \mathbb{R}_{+}^{n \times n}$ sehingga mengakibatkan $(F+T \mathcal{K})^{-1} S \in \mathbb{R}_{+}^{n \times n}$. Selain itu, dari kondisi (c) dan (d) mengakibatkan $(F+$ $T \mathcal{K})^{-1} T \in \mathbb{R}_{+}^{n \times n}$. Sehingga sistem (1.3) adalah positif.

Contoh 3.3. Diberikan sistem (1.1) dengan

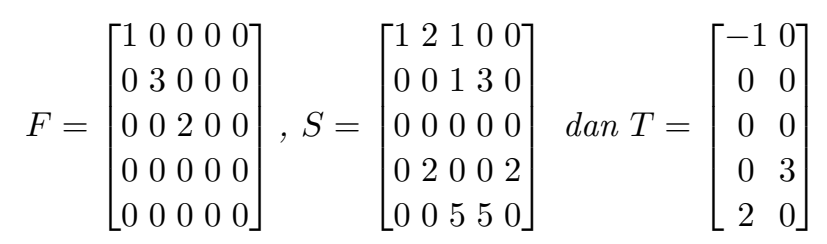

Misalkan

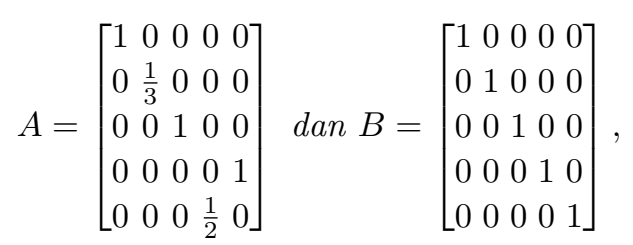

maka diperoleh

$$
A F B=\left[\begin{array}{lllll}
1 & 0 & 0 & 0 & 0 \\
0 & 1 & 0 & 0 & 0 \\
0 & 0 & 2 & 0 & 0 \\
0 & 0 & 0 & 0 & 0 \\
0 & 0 & 0 & 0 & 0
\end{array}\right] \text { dan } A T=\left[\begin{array}{cc}
-1 & 0 \\
0 & 0 \\
0 & 0 \\
2 & 0 \\
0 & \frac{3}{2}
\end{array}\right]
$$

Selain itu

$$
\begin{gathered}
T_{1} T_{2}^{T}=\left[\begin{array}{cc}
-2 & 0 \\
0 & 0 \\
0 & 0
\end{array}\right] \in \mathbb{R}_{-}^{3 \times 2},\left(T_{2} T_{2}^{T}\right)^{-1}=\left[\begin{array}{cc}
\frac{1}{4} & 0 \\
0 & \frac{4}{9}
\end{array}\right] \in \mathbb{R}_{+}^{2 \times 2}, \\
T_{1}-T_{1} T_{2}^{T}\left(T_{2} T_{2}^{T}\right)^{-1} T_{2}=\left[\begin{array}{ll}
1 & 0 \\
0 & 0 \\
0 & 0
\end{array}\right] \in \mathbb{R}_{+}^{3 \times 2},\left(T_{2} T_{2}^{T}\right)^{-1} T_{2}=\left[\begin{array}{cc}
\frac{1}{2} & 0 \\
0 & \frac{2}{3}
\end{array}\right] \in \mathbb{R}_{+}^{2 \times 2} .
\end{gathered}
$$

Pilih matriks $\mathcal{K}=\left[\begin{array}{lllll}0 & 0 & 0 & 2 & 0 \\ 0 & 0 & 0 & 0 & \frac{3}{2}\end{array}\right]$ maka

$$
\operatorname{det}(F+T \mathcal{K})=\operatorname{det}\left[\begin{array}{ccccc}
1 & 0 & 0 & -2 & 0 \\
0 & 3 & 0 & 0 & 0 \\
0 & 0 & 2 & 0 & 0 \\
0 & 0 & 0 & 0 & \frac{9}{2} \\
0 & 0 & 0 & 4 & 0
\end{array}\right] \neq 0
$$


68 Rakib Hablullah, Zulakmal

Selanjutnya

$$
(F+T \mathcal{K})^{-1} S=\left[\begin{array}{ccccc}
1 & 2 & \frac{7}{2} & \frac{5}{2} & 0 \\
0 & 0 & \frac{1}{3} & 1 & 0 \\
0 & 0 & 0 & 0 & 0 \\
0 & 0 & \frac{5}{4} & \frac{5}{4} & 0 \\
0 & \frac{4}{9} & 0 & 0 & \frac{4}{9}
\end{array}\right] \in \mathbb{R}_{+}^{5 \times 5},(F+T \mathcal{K})^{-1} T=\left[\begin{array}{ll}
0 & 0 \\
0 & 0 \\
0 & 0 \\
\frac{1}{2} & 0 \\
0 & \frac{2}{3}
\end{array}\right] \in \mathbb{R}_{+}^{5 \times 2} .
$$

Sehingga sistem (1.3) merupakan sistem normal positif.

\section{Daftar Pustaka}

[1] Anton, H. 1991. Aljabar Linier Elementer. Edisi Kedelapan-Jilid 1. Erlangga. Jakarta.

[2] Campbell S. L. 1980. Singular system of differential equatins. Pitman Books Ltd, London.

[3] Canto,B., C. Coll and E. Sanches. 2006. Positive Normalizable Singular System. Departement de Matematic Aplicada, Universitat Politeenica de Valencia, 46071 Valencia, Spain.

[4] Dai, L 1989. Singular control system. Springer-Verlag, Berlin Heidelberg New York.

[5] Farina L, Rinaldi S. 2000. Positive linier system. Jhon Wiley and Sons, New York.

[6] G.-R. Duan. 2010. Analysis and Design of Descriptor Linier System. Springer, New York.

[7] Kaczorek, T. 2002. Positive 1D and 2D system. Springer, London.

[8] Minc, H. 1988. Nonnegative matrices. John Wiley and Sons, New York.

[9] Rina, I. 2015. Realisasi Positif Stabil Asimtotik Dari Sistem Linier Diskrit Dengan Pole Konjugat Kompleks. Universitas Andalas, Padang. 